\title{
Efecto Antimetastásico de Celecoxib/PLGA en un Modelo Murino de Adenocarcinoma Mamario TA3-MTX-R
}

\author{
Antimetastatic Effect of Celecoxib/PLGA in a TA3-MTX-R Murine Breast Adenocarcinoma Model
}

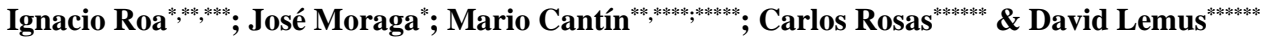

ROA, I.; MORAGA, J.; CANTIN, M.; ROSAS, C. \& LEMUS, D. Efecto antimetastásico de Celecoxib/PLGA en un modelo murino de adenocarcinoma mamario TA3-MTX-R. Int. J. Morphol., 33(2):558-565, 2015.

RESUMEN: La metástasis es el proceso de propagación de un foco cancerígeno a un órgano distinto de aquel en que se inició; ocurriendo generalmente por vía sanguínea o linfática. La localización más frecuentes de las metástasis son los órganos más irrigados como el cerebro, pulmones, hígado, huesos y glándulas suprarrenales. El objetivo fue analizar el patrón de metástasis hepática de tumor TA3-MTX-R, luego de la aplicación del antiangiogénico Celecoxib microencapsulado en PLGA en ratones, así como la disminución de áreas metastásicas a nivel lobulillar. Se utilizó un modelo de tumor experimental, inducido por células TA3-MTX-R, en 18 ratones, separados en 3 grupos de 6 animales, los cuales fueron tratados con dos presentaciones de Celecoxib en administración intramuscular (Grupo 1, Control: TA3-MTX-R; Grupo 2: TA3-MTX-R+Cx y Grupo 3: TA3-MTX-R+Cx/PLGA). Los ratones fueron sacrificados y procesados histológicamente para ser teñidos con H\&E y Tricrómico de Arteta. El estudio reveló que el higado muestra una marcada heterogeneidad, y un patrón de metástasis perivascular y neovascularización central y periférica. Además, Celecoxib redujo significativamente la invasión tumoral en el hígado ( $\mathrm{p}<0,0001)$. Los resultados son similares a descripciones parciales realizadas previamente y son comparables a otras líneas tumorales. Creemos que la vía de administración del fármaco es crítica para la interpretación de los resultados. Los hallazgos son importantes para la discusión de otras investigaciones en donde Celecoxib es usado como un fármaco antiangiogénico.

PALABRAS CLAVE: Metástasis; Hígado; TA3-MTX; Celecoxib; PLGA.

\section{INTRODUCCIÓN}

La metástasis es el proceso de propagación de un foco canceroso a un órgano distinto de aquel en que se inició; ocurriendo generalmente por vía sanguínea o linfática y son la causa de aproximadamente el $98 \%$ de las muertes por cánceres no detectados. Las localizaciones más frecuentes de las metástasis son los órganos más irrigados tales como son cerebro, pulmones, hígado, huesos y glándulas suprarrenales (Khanfir et al., 2013).

Uno de los acontecimientos críticos requeridos para el crecimiento de los tumores y la producción de sus metástasis es el desarrollo de una nueva red de los vasos sanguíneos (angiogénesis) (Roa, 2014). Las terapias antitumorales actuales buscan inhibir dicho evento, para lo cual se han comenzado a emplear fármacos inhibidores selectivos de la ciclooxigensa 2 (COX-2), tales como el celecoxib (Rosas et al., 2013).

COX-2 se encuentra sobrexpresada en distintos tipos de cáncer (Ho et al., 2014) y su bloqueo por parte del celecoxib juega un rol importante en la disminución de la metástasis hematógena del cáncer colorectal en hígado (Chen et al., 2001; Kakiuchi et al., 2002; Ninomiya et al., 2012). Celecoxib ha mostrado además, la capacidad de bloquear la angiogenesis e inhibir el crecimiento de cáncer de colon y pulmón en ratones (Masferrer et al., 2000).

Si bien los efectos del celecoxib son favorables, su administración oral durante tiempos prolongados causarían complicaciones tales como toxicidad sistémica (Bombardier

* Unidad de Morfología, Departamento de Ciencias Básicas Biomédicas, Facultad de Ciencias de la Salud, Universidad de Talca, Chile.

** Programa de Doctorado en Morfología, Facultad de Medicina, Universidad de La Frontera, Temuco, Chile.

**** Becario CONICYT-PCHA/Doctorado Nacional/2015-21150235

***** CEMyQ, Facultad de Medicina, Universidad de La Frontera, Temuco, Chile.

****** Centro de Investigación Biomédica, Universidad Autónoma de Chile, Chile

******* Laboratorio de Embriología Experimental y Molecular, Programa de Anatomía y Biología del Desarrollo, Instituto de Ciencias Biomédicas, Facultad de Medicina, Universidad de Chile, Chile. 
et al., 2000) y problemas gastrointestinales (Silverstein et $a l ., 2000$ ) o daño y eventos cardiovasculares (Caldwell et al., 2006). Otros estudios indican que la administración frecuente en animales de experimentación de altas dosis de celecoxib oral, causaría infiltración mononuclear, hiperplasia y degeneración a nivel renal, además de pérdida de algunas funciones hepáticas (Koçkaya et al., 2010).

Con el fin de mejorar la biodisponibilidad de los fármacos y disminuir sus posibles efectos adversos, es que estos han sido asociados con ácido poli(lactico-co-glycolico) (PLGA) (Vilos et al., 2014, 2015), donde en formulaciones de nano y micropartículas han sido usadas en conjunto con múltiples agentes antiangiogénicos en terapias antitumorales (Anand et al., 2010; Qi et al., 2011), tales como el celecoxib (Amrite et al., 2006).

Dado que ha sido relatada la efectividad de la acción antitumoral del Celecoxib y que han sido descritos múltiples efectos adversos en su utilización en altas dosis por largo tiempo, es necesario evaluar nuevas vías de administración tales como las otorgadas gracias a la microencapsulación con PLGA. El motivo principal del presente estudio es comprobar la efectividad en la disminución de la metástasis, del Celecoxib microencapsulado en PLGA, en tumor sólido inducido por células tumorales TA3-MTX$\mathrm{R}$ en ratones (Mus musculus), así como una descripción histológica detallada de la metástasis hepatica.

\section{MATERIAL Y MÉTODO}

Línea Tumoral. Se utilizaron células tumorales de adenocarcinoma mamario TA3 en su variedad resistente a metotrexato (TA3-MTX-R) de crecimiento ascítico, provenientes del Laboratorio de Bioenergótica y Cáncer, Programa de Farmacología Molecular y Clínica, Facultad de Medicina, Universidad de Chile. Las células TA3-MTX-R fueron seleccionadas a partir de un tumor ascítico TA3 in vivo, a través de traspasos sucesivos de fluido ascítico en ratones, los cuales fueron tratados por vía peritoneal con metotrexato (MTX). La dosis de MTX fue aumentada con cada traspaso, de 0,1 a $2,5 \mathrm{mg} / \mathrm{kg} / 48 \mathrm{~h}$. Esta línea tumoral es resistente además a Vinblastina, 5-Fluoruracilo, Cisplatino y CARFENISOP. La obtención de las células tumorales de la línea TA3-MTX-R se realizó al sexto día post-inoculación intraperitoneal de células tumorales contenidas en el fluido ascítico de ratones de la cepa AJ. Una vez eutanasiados, se obtuvo $2 \mathrm{~mL}$ de líquido ascítico y se diluyeron en $8 \mathrm{~mL}$ de suero fisiológico. Se realizó un conteo en cámara de Neubauer, donde se obtuvo una concentración final de 2 ×10 células por $\mathrm{mL}$ de la suspensión tumoral. Para verificar la vitalidad de las células tumorales se tomaron $25 \mu \mathrm{L}$ de la suspensión de células y se mezclaron con $25 \mu \mathrm{L}$ de Azul de Trypan $0,5 \%$, debiendo obtener una concentración igual o superior al $95 \%$, que es el requerido para un crecimiento tumoral óptimo.

Fármaco . Celecoxib: Fue utilizado Celecoxib 200 mg (Celebra ${ }^{\circledR}$, Pfizer@), el cual fue diluido en el H20 destilada hasta alcanzar la concentración media de 1000 ppm, según lo descrito por Ragel et al. (2007).

Celecoxib/PLGA: Fue encapsulado Celecoxib $200 \mathrm{mg}$ (Celebra®, Pfizer@), en microcápsulas de PLGA de acuerdo a lo descrito por Ayalasomayajula \& Kompella (2005), en la cual se obtuvo una concentración media de 1000 ppm. Se utilizó PLGA (poli (D, L-láctico-co-glicólico)) con una proporción de monómero 50:50, de terminación éster y viscosidad de 0,15-0,24, 0,26-0,54 y 0,55-0,75 dl/g, (Lactel Absorbibles Polymers, Pelham, Alabama, EE.UU.). El poli (vinil-alcohol) (PVA) fue hidrolizado un $87-90 \%$, con un peso promedio 30,000-70,000 mol (Sigma-Aldrich, St. Louis, MO, EE.UU.). Las micropartículas (MPs) de PLGA fueron preparadas por método de emulsión de evaporación del disolvente agua-aceite-agua (w/o/w). Una solución de 1 $\mathrm{mL}$ de agua doble destilada fue emulsificada en $5 \mathrm{~mL}$ de DCM que contiene $100 \mathrm{mg}$ de PLGA, utilizando un homogeneizador de alta velocidad a $35.000 \mathrm{rpm}$ (Thermo Fisher Scientific, Waltham, MA) de sistema Ultra-Turrax. En esta primera emulsión (w/o) se agregó a $50 \mathrm{ml}$ de PVA al 1\% ( $1 \mathrm{mg} / \mathrm{mL}$ ) y fue emulsionada con el mismo sistema. En la emulsión final (w/o/w) se procedió a mezclar con $100 \mathrm{ml}$ de poli vinil alcohol (PVA) a $0,01 \%$. Las micropartículas fueron recolectadas por filtración. Una vez tamizadas, las microesferas fueron liofilizadas y almacenadas a $-20{ }^{\circ} \mathrm{C}$. Se obtuvieron micropartículas de entre 0,5 y $10 \mu \mathrm{m}$ de diámetro.

Material Biológico. Fue utilizado un modelo de tumor experimental, inducido por células TA3-MTX-R, en 18 ratones, separados en 3 grupos de 6 animales, los cuales fueron tratados con dos presentaciones de Celecoxib en administración intramuscular (Grupo 1, Control: TA3-MTX-R; Grupo 1: TA3-MTX-R+Cx y Grupo 3: TA3-MTX-R+Cx/ PLGA). Fueron mantenidos ad libitum, en jaulas conservadas a temperatura ambiente y de acuerdo a las normas impuestas por el Comité de Bioética de la Facultad de Medicina de la Universidad de Chile (CBA 0498 FMUCH).

Las muestras de los hígados fueron fijados en Formalina Tamponada al $10 \%$ por $48 \mathrm{~h}$, realizándose el procesamiento histológico necesario para obtener cortes de 5 $\mathrm{mm}$, los que fueron teñidos con H\&E y Tricrómico de Arteta, según lo descrito por Rosas et al. (2013). Se agregaron muestras de hígado sano de ratón, como control 
ROA, I.; MORAGA, J.; CANTIN, M.; ROSAS, C. \& LEMUS, D. Efecto antimetastásico de Celecoxib/PLGA en un modelo murino de adenocarcinoma mamario TA3-MTX-R Int. J. Morphol., 33(2):558-565, 2015.

Estimación de la invasión tumoral y análisis estadísticos. Para la comparación de la invación tumoral del hígado se realizó una estimación cuantitativa de un campo microscópico de acuerdo a la presencia de células tumorales utilizando el programa ImageJ $1.47 \mathrm{v}$ para Windows; de la cual se calculó el área metastásica (AM) correspondiente en cada imagen. Para la lectura histológica de las preparaciones se utilizó un microscopio óptico Trinocular PrimoStar (Carl Zeiss, Göttingen, Germany). Las imágenes fueron obtenidas con una cámara digital EOS Rebel XSI (Canon Inc. Japan) acoplada al microscopio y conectada a un computador para las mediciones.

La estimación por grupo se realizó observando 30 campos microscópicos con la técnica de doble enmascaramiento; registrándose la concordancia utilizando coeficiente kappa para las características cualitativas y coeficiente de Lin para las características cuantitativas, tabulando los datos de acuerdo a lo observado en cada uno de ellos. Se calculó el promedio, error estándar y se aplicó el la prueba $t$ de Student.

\section{RESULTADOS}

La metástasis hepática de células TA3-MTX-R, presenta marcada heterogeneidad y un patrón de metástasis perivascular.

Histológicamente, los hígados de los ratones del grupo inoculado con células TA3-MTX-R, presentaron un parénquima hepático afectado abundantemente, mostrando en diversas áreas; desplazamiento de las columnas de hepatocitos que fueron reemplazadas por nidos de células, las cuales se ubicaron alrededor de vasos sanguíneos como las venas centrolobulillares y de los espacios porta. Además en los sinusoides hepáticos, se podía observar una gran cantidad de células además de eritrocitos. La morfología de las células observadas era variada, encontrando polimorfonucleares, linfocitos y células compatibles con células tumorales (CT). Esta invasión celular provocó una desorganización de la arquitectura lobulillar característica del hígado (Fig. 1).

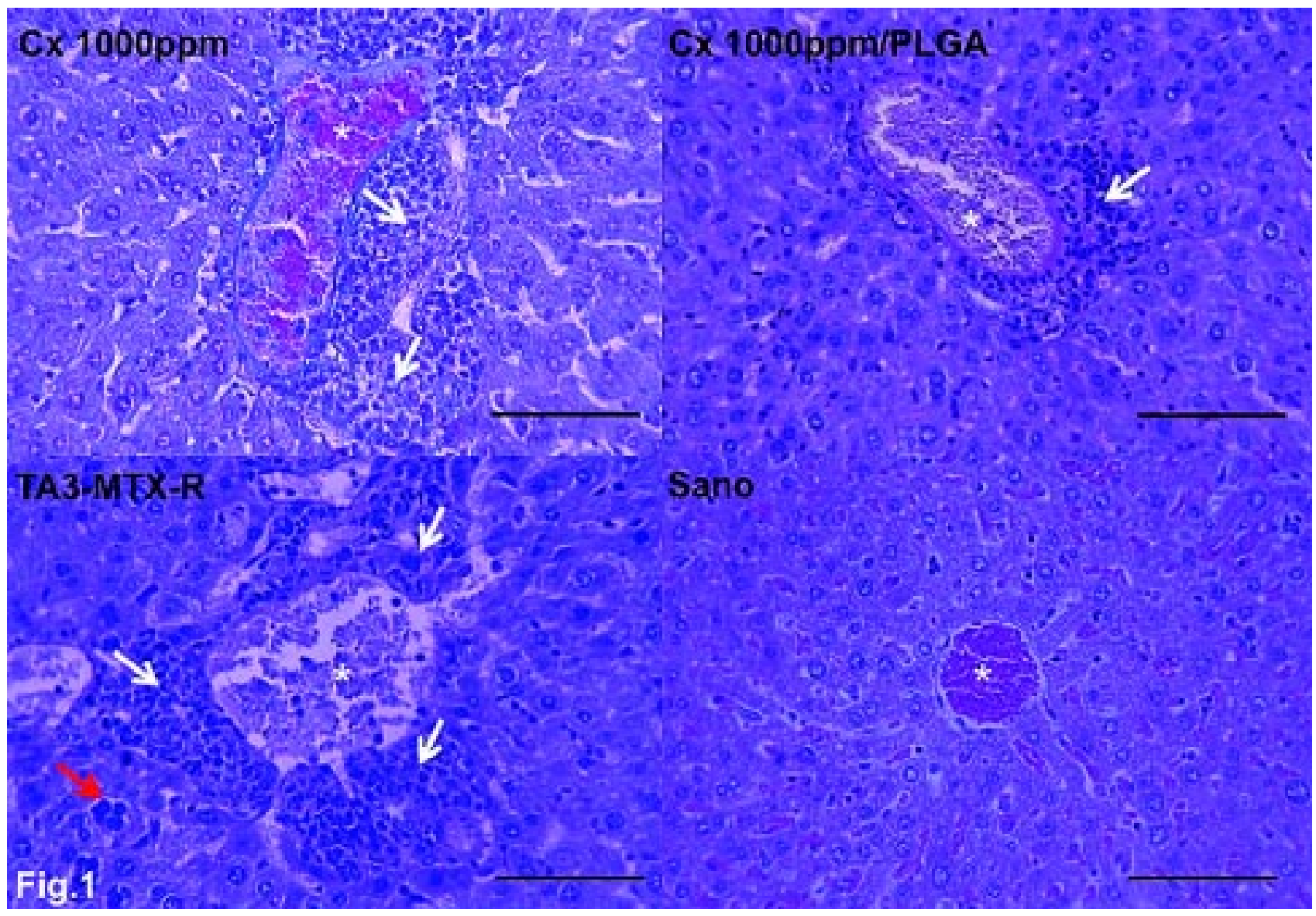

Fig. 1. Aspecto histológico de hígado de ratones. Células tumorales (flecha blanca) en torno a los elementos vasculares, vena centro lobulillar (asterisco). Células tumorales (flecha roja) entre hepatocitos. Hematoxilina \& Eosina (400x); barra 100 mm. 
GRUPO 2 TA3-MTX-R/Cx: El parénquima hepático se vio moderadamente afectado; se observaron nidos de CT que estaban alrededor de vasos sanguíneos; tales como venas centrolobulillares y espacios porta. En la zona de los sinusoides hepáticos, se pudo observar eritrocitos, además de polimorfonucleares, linfocitos (Fig. 1).

GRUPO 3 TA3-MTX-R/CxPLGA: El parénquima hepático se vio levemente afectado, presentando en algunas áreas de infiltración las cuales no alteraron la morfología de las columnas de hepatocitos. Se aprecian además algunos pequeños nidos de células alrededor de vasos sanguíneos centrolobulillares y de los espacios porta, los cuales además presentaban polimorfonucleares y linfocitos (Fig. 1).

En general, las muestras de los órganos de ratones inoculados con células tumorales, se caracterizaron por presentar una invasión celular principalmente en aquellos órganos altamente vascularizados como es el caso del pulmón

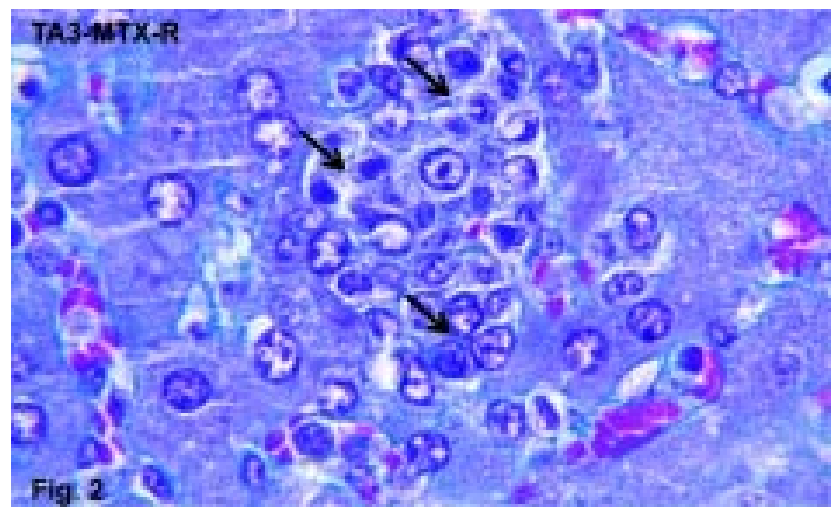

Fig. 2. Aspecto histológico de hígado de ratón TA3-MTX-R. Células mononucleares, polimorfonucleares y células tumorales (flechas). Hematoxilina \& Eosina (1000x).

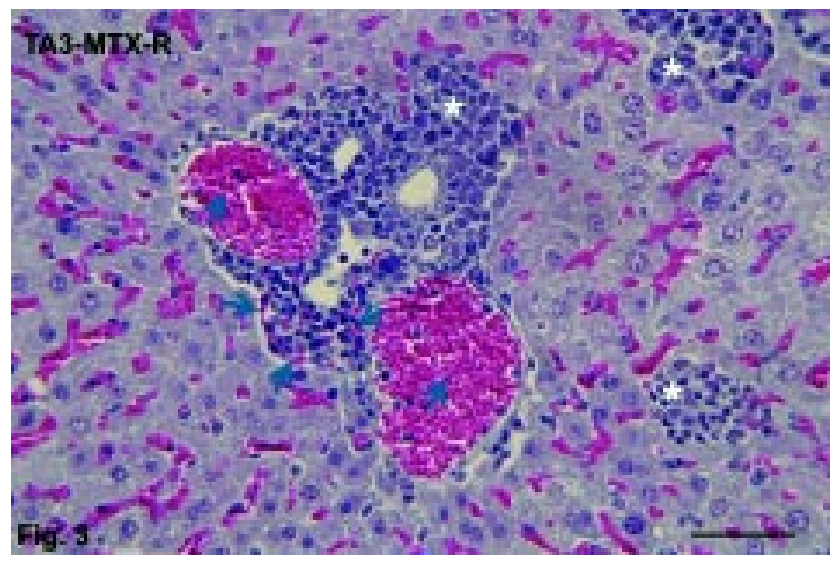

Fig. 3. Aspecto histológico de hígado de ratón TA3-MTX-R. Células células tumorales (asterisco) en torno a nuevos elementos vasculares (flechas). Tricrómico de Arteta (400x); barra 100 mm. (datos no mostrados) y el hígado. Las células más características de este proceso fueron las células mononucleares, polimorfonucleares y células tumorales (Fig. 2). Junto al infiltrado celular se observó una abundancia en la vascularización en sitios cercanos a los nidos celulares descritos (Fig. 3).

Celecoxib microencapsulado en PLGA y Celecoxib no encapsulado, generan una disminución de áreas metastásicas en hígados de ratones inoculados con células TA3-MTX-R.

Al comparar la metástasis de las células tumorales TA3-MTX-R, en hígado de ratones tratados con Celecoxib microencapsulado en PLGA y Celecoxib no encapsulado, el grupo control presento mayor área metastásicas que los grupos con tratamiento con Celecoxib; diferencia que fue estadísticamente significativa ( $<<0,0001)$ (Fig. 4).

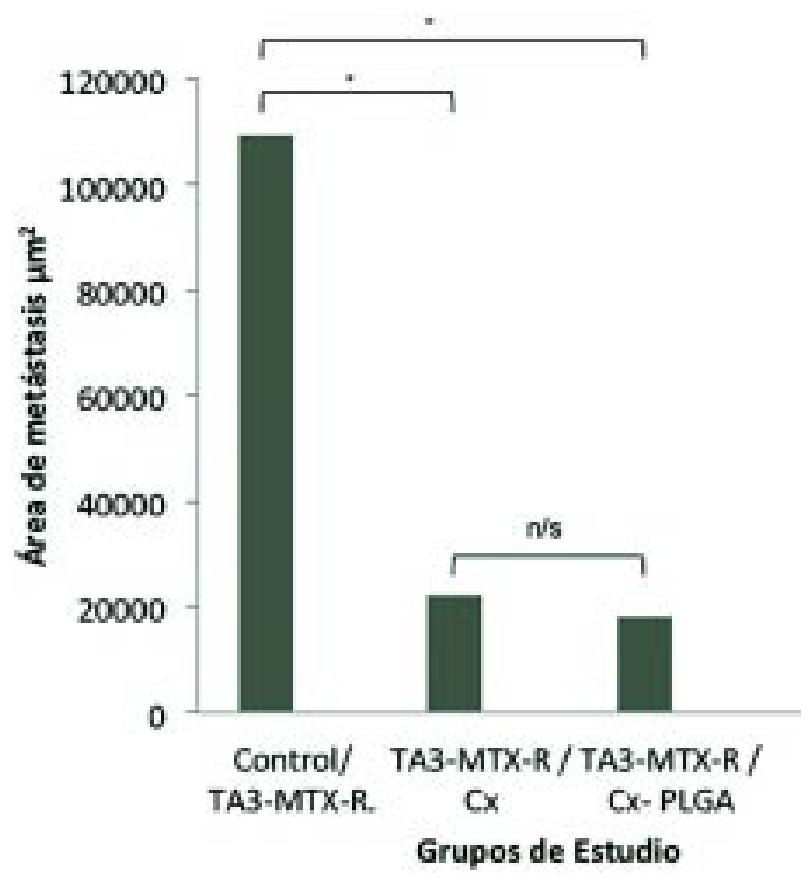

Fig. 4. Densidad de áreas metastásicas del tumor TA3-MTX-R por grupo de estudio.

\section{DISCUSIÓN}

El crecimiento de los tumores sólidos y la formación de metástasis posteriores dependen de la generación de nuevos vasos; es así como las células tumorales inducen este proceso, al producir numerosos factores proangiogénicos 
(Gavalas et al., 2013). Las terapias antiangiogénicas actuales buscan inhibirlos con el fin de disminuir la neovascularización, apoptosis y metástasis. Estos puede ser suprimidos por múltiples agentes entre ellos los inhibidores selectivos de la COX-2, tal como el celecoxib, importante agente anti-inflamatorio (Venkatesan et al., 2011), que se encuentra bajo estudio para el tratamiento de variados tumores malignos tales como cáncer colorectal, cáncer de mama, pulmón y próstata (Amrite et al.).

Existe evidencia de sobreexpresión de COX-2 en una variedad de cánceres (Cianchi et al., 2001; Dempke et al., 2001), además sería para algunos autores la responsable del aumento en el crecimiento tumoral y su rápida proliferación. Estudios confirman que pacientes que sobreexpresan $\mathrm{COX}-2$ en células tumorales pancreáticas tienen peor pronóstico que aquellos que no lo hacen (Ragel et al.; Xu et al., 2008).

La sobreexpresión de COX-2 en células tumorales afecta la angiogénesis y por tanto la metástasis por la producción de eicosanoides (TAX2; PGI2 y PGE2) (Wang $e t$ al., 2013), los cuales estimulan la migración de células endoteliales y angiogénesis por el incremento en la expresión de VEGF estimulando la proliferación de células endoteliales.

En el presente estudio, celecoxib redujo la metástasis de celulas TA3-MTX-R, en hígado, tanto en su presentación asociada con PLGA, como no microencapsulada. Si bien ha sido previamente reportado su acción frente a metástasis pulmonar (Gendy et al., 2011; Barlow et al., 2012; Zhang et al., 2014) y hueso (Das Roy et al., 2009), y frente a apaptosis y crecimiento tumoral en el mismo modelo (Rosas et al., 2014); no hay información de su efecto frente a metástasis cuando es asociado a micropartículas de PLGA.

La asociación a PLGA ha sido reportada previamente, con una reducción significativa de los niveles de VEGF y de PGE2, en un modelo de tumor pulmonar en ratones de la cepa AJ, así como celecoxib/PLGA también ha sido utilizada en terapias antiangiogénicas de patologías oculares (Amrite et al.) y otros tratamientos antitumorales (Ibrahim et al., 2013), con efectos favorables como la disminución de la angiogénesis e inducción de la apoptosisde las células tumorales (Cui et al., 2010).

El celecoxib inhibe la vía de la síntesis de prostaglandinas y hace más lenta la progresión del cáncer, al menos en parte mediante la reducción de las propiedades inmunosupresoras y angiogénicas de PGE2 (Li et al., 2013; Perroud et al., 2013). Además de estas propiedades, PGE2 puede estimular directamente las vías de supervi- vencia/metástasis de células cancerosas por medio de PI3K, Ras o Wnt/ $\beta$-catenina (Zhou et al., 2005; Wang \& Dubois, 2010).

Hallazgos recientes sugieren que celecoxib también puede tener un impacto sobre el metabolismo del tumor, y puede limitar el crecimiento del tumor, en parte, mediante la reducción intracelular de citrato (Ho et al.).

La acción antimetastásica del Celecoxib asociado a PLGA no ha sido reportada previamente, así como una descripción histológica detallada de la metástasis frente a la acción de este. Es por ello que el estudio de este fármaco en un modelo in vivo resistencia a drogas antineoplásicas como el tumor TA3-MTX-R es importante para comprender la biología del cáncer.

Las características del tumor observado en nuestros resultados se presentan muy similares a las relatadas por Rosas et al. (2013), en donde el tipo celular, su morfología, ubicación y áreas del tumor fueron similares, al utilizar la misma línea tumoral TA3-MTX-R. Por su parte, otras líneas tumorales mamarias tales como la SCK se describen muy similares en cuanto a vascularización, invasividad y generación de metástasis cuando son comparados con la línea TA3-MTX-R (Farooqui et al., 2007).

Con respecto a las vías de diseminación tumoral, Kumar et al. (2005) describen la existencia de una hematógena que invade a principalmente hígado y pulmones, coincidente a lo encontrado en nuestro estudio y relatado por Labelle \& Hynes (2012). En el caso de la metástasis hepática, Kumar et al. describieron que este órgano se ve afectado principalmente por las neoplasias de mama, pulmón y colon, en donde existen área de necrosis central, hepatomegalia y nodularidad del margen libre. Esto fue observado parcialmente en los resultados ya que si bien la línea tumoral corresponde a una línea mamaria, no se observó hepatomegalia, la nodularidad ni la necrosis central, sólo hallando una mayor invasión en la periferia de los vasos sanguíneos intrahepáticos, similar a lo reportado por Rosas et al. (2013).

Se observó una disminución en la invasión de células tumorales, cuando fue aplicado el celecoxib/PLGA y en menor grado cuando se aplicó celecoxib no asociado a PLGA, con estos resultados reforzamos la idea del uso de fármacos que permitan la inhibición de varias características de la progresión tumoral como es el caso de la angiogénesis y metástasis y establecer así las bases para futuras investigaciones asociadas a esta línea tumoral (Peng et al., 2013). 
Celecoxib logra ser una buena alternativa en la terapia antiangiogénica y por ende antimetastásico. La vía de administración podría jugar un rol importante en el proceso de inhibición de la metástasis, por lo cual la exploración de nuevas vías y asociaciones de fármacos favorecerían la investigación en el área de la oncología.

\section{CONCLUSIÓN}

Celecoxib asociado a PLGA o a otro tipo de polímero son vías factibles de administración de fármacos, ya que podrían disminuir la frecuencia de administración, lo que lleva a una mayor aceptación por parte del paciente; así como un aumento del beneficio terapéutico, debido a la elimina- ción de las fluctuaciones en los niveles séricos del fármaco. La potencial disminución de la dosis total requerida para un tratamiento, debido a una mayor eficiencia en el aprovechamiento de la dosis administrada y la potencial disminución de los efectos adversos

\section{AGRADECIMIENTOS}

A la Sra. Irma Orellana, por su apoyo técnico. Al Dr. Jorge Ferreira y al Sr. Jorge Leiva del Laboratorio de Bioenergética y Cáncer, Programa de Farmacología Molecular y Clínica, Facultad de Medicina, Universidad de Chile. Dr. Cristian Vilos, del Centro para el Desarrollo de la Nanociencia y Nanotecnología, Universidad de Santiago, Chile.

ROA, I.; MORAGA, J.; CANTIN, M.; ROSAS, C. \& LEMUS, D. Antimetastatic effect of Celecoxib/PLGA in a TA3-MTX-R murine breast adenocarcinoma model. Int. J. Morphol., 33(2):558-565, 2015.

SUMMARY: Metastasis is the propagation process of a cancerous focus to an organ other than that in which it started; usually occurring through blood or lymphatic route. The most common sites of metastases are the organs most irrigated such as the brain, lungs, liver, bones and adrenal glands. The objective was to analyze the pattern of liver tumor metastasis TA3-MTX-R, after application of antiangiogenic Celecoxib microencapsulated in PLGA in mice and decreased metastatic to lobular level areas. An experimental model of tumor induced TA3-MTX-R cells was used, 18 mice divided into 3 groups of 6 animals, which were treated with two presentations Celecoxib intramuscular (Group 1, control was used -R; Group 1: TA3-MTX-R+Cx and Group 3: TA3-MTX-R+Cx/PLGA). The mice were sacrificed and processed histologically to be stained with H\&E and Arteta trichrome. The study revealed that the liver shows a marked heterogeneity, and a pattern of perivascular metastasis and central and peripheral neovascularization. Furthermore, Celecoxib significantly reduced tumor invasion in the liver $(p<0.0001)$. The results are similar to partial descriptions made previously and are comparable to other tumor lines. It is believed that the route of administration of the drug is critical for the interpretation of the results. These are important for the discussion of other investigations in which Celecoxib is used as an antiangiogenic drug.

KEY WORDS: Metastasis; Liver; TA3-MTX; Celecoxib; PLGA.

\section{REFERENCIAS BIBLIOGRÁFICAS}

Amrite, A. C.; Ayalasomayajula, S. P.; Cheruvu, N. P. \& Kompella, U. B. Single periocular injection of celecoxib-PLGA microparticles inhibits diabetes-induced elevations in retinal PGE2, VEGF, and vascular leakage. Invest. Ophthalmol. Vis. Sci., 47(3):1149-60, 2006.

Anand, P.; Nair, H. B.; Sung, B.; Kunnumakkara, A. B.; Yadav, V. R.; Tekmal, R. R. \& Aggarwal, B. B. Design of curcuminloaded PLGA nanoparticles formulation with enhanced cellular uptake, and increased bioactivity in vitro and superior bioavailability in vivo. Biochem. Pharmacol., 79(3):330-8, 2010 .

Ayalasomayajula, S. P. \& Kompella, U. B. Subconjunctivally administered celecoxib-PLGA microparticles sustain retinal drug levels and alleviate diabetes-induced oxidative stress in a rat model. Eur. J. Pharmacol., 511(2-3):191-8, 2005.
Barlow, M.; Edelman, M.; Glick, R. D.; Steinberg, B. M. \& Soffer, S. Z. Celecoxib inhibits invasion and metastasis via a cyclooxygenase 2 -independent mechanism in an in vitro model of Ewing sarcoma. J. Pediatr. Surg., 47(6):1223-7, 2012.

Bombardier, C.; Laine, L.; Reicin, A.; Shapiro, D.; BurgosVargas, R.; Davis, B.; Day, R.; Ferraz, M. B.; Hawkey, C. J.; Hochberg, M. C.; Kvien, T. K.; Schnitzer, T. J. \& VIGOR Study Group. Comparison of upper gastrointestinal toxicity of rofecoxib and naproxen in patients with rheumatoid arthritis. VIGOR Study Group. N. Engl. J. Med., 343(21):1520-8, 2000 .

Caldwell, B.; Aldington, S.; Weatherall, M.; Shirtcliffe, P. \& Beasley, R. Risk of cardiovascular events and celecoxib: a systematic review and meta-analysis. J. R. Soc. Med., 99(3):132-40, 2006. 
Chen, W. S.; Wei, S. J.; Liu, J. M.; Hsiao, M.; Kou-Lin, J. \& Yang, W. K. Tumor invasiveness and liver metastasis of colon cancer cells correlated with cyclooxygenase-2 (COX-2) expression and inhibited by a COX-2-selective inhibitor, etodolac. Int. J. Cancer, 91(6):894-9, 2001.

Cianchi, F.; Cortesini, C.; Bechi, P.; Fantappiè, O.; Messerini, L.; Vannacci, A.; Sardi, I.; Baroni, G.; Boddi, V.; Mazzanti, R. \& Masini, E. Up-regulation of cyclooxygenase 2 gene expression correlates with tumor angiogenesis in human colorectal cancer. Gastroenterology, 121(6):1339-47, 2001.

Cui, F. Y.; Song, X. R.; Li, Z. Y.; Li, S. Z.; Mu, B.; Mao, Y. Q.; Wei, Y. Q. \& Yang, L. The pigment epithelial-derived factor gene loaded in PLGA nanoparticles for therapy of colon carcinoma. Oncol. Rep., 24(3):661-8, 2010.

Das Roy, L.; Pathangey, L. B.; Tinder, T. L.; Schettini, J. L.; Gruber, H. E. \& Mukherjee, P. Breast-cancer-associated metastasis is significantly increased in a model of autoimmune arthritis. Breast Cancer Res., 11(4):R56, 2009.

Dempke, W.; Rie, C.; Grothey, A. \& Schmoll, H. J. Cyclooxygenase2: a novel target for cancer chemotherapy? J. Cancer Res. Clin. Oncol., 127(7):411-7, 2001.

Farooqui, M.; Li, Y.; Rogers, T.; Poonawala, T.; Griffin, R. J.; Song, C. W. \& Gupta, K. COX-2 inhibitor celecoxib prevents chronic morphine-induced promotion of angiogenesis, tumour growth, metastasis and mortality, without compromising analgesia. $\mathrm{Br}$. J. Cancer, 97(11):1523-31, 2007.

Gavalas, N. G.; Liontos, M.; Trachana, S. P.; Bagratuni, T.; Arapinis, C.; Liacos, C.; Dimopoulos, M. A. \& Bamias, A. Angiogenesisrelated pathways in the pathogenesis of ovarian cancer. Int. J. Mol. Sci., 14(8):15885-909, 2013.

Gendy, A. S.; Lipskar, A.; Glick, R. D.; Steinberg, B. M.; Edelman, M. \& Soffer, S. Z. Selective inhibition of cyclooxygenase-2 suppresses metastatic disease without affecting primary tumor growth in a murine model of Ewing sarcoma. J. Pediatr. Surg., 46(1):108-14, 2011.

Ho, V. W.; Hamilton, M. J.; Dang, N. H.; Hsu, B. E.; Adomat, H. H.; Guns, E. S.; Weljie, A.; Samudio, I.; Bennewith, K. L. \& Krystal, G. A low carbohydrate, high protein diet combined with celecoxib markedly reduces metastasis. Carcinogenesis, 35(10):2291-9, 2014.

Ibrahim, M. M.; Abd-Elgawad, A. E.; Soliman, O. A. \& Jablonski, M. M. Nanoparticle-based topical ophthalmic formulations for sustained celecoxib release. J. Pharm. Sci., 102(3):1036-53, 2013.

Kakiuchi, Y.; Tsuji, S.; Tsujii, M.; Murata, H.; Kawai, N.; Yasumaru, M.; Kimura, A.; Komori, M.; Irie, T.; Miyoshi, E.; Sasaki, Y.; Hayashi, N.; Kawano, S. \& Hori, M. Cyclooxygenase-2 activity altered the cell-surface carbohydrate antigens on colon cancer cells and enhanced liver metastasis. Cancer Res., 62(5):156772, 2002.
Khanfir, A.; Lahiani, F.; Bouzguenda, R.; Ayedi, I.; Daoud, J. \& Frikha, M. Prognostic factors and survival in metastatic breast cancer: A single institution experience. Rep. Pract. Oncol. Radiother, 18(3):127-32, 2013.

Koçkaya, E. A.; Selmanog `lu, G.; Kismet, K. \& Akay, M. T. Pathological and biochemical effects of therapeutic and supratherapeutic doses of celecoxib in Wistar albino male rats. Drug Chem. Toxicol., 33(4):410-4, 2010.

Kumar, V.; Abbas, A. K. \& Fausto, N. Robbins y Cotran: Patología Estructural y Funcional. $7^{\mathrm{a}}$ ed. Madrid, Elsevier, 2005.

Masferrer, J. L.; Leahy, K. M.; Koki, A. T.; Zweifel, B. S.; Settle, S. L.; Woerner, B. M.; Edwards, D. A.; Flickinger, A. G.; Moore, R. J. \& Seibert, K. Antiangiogenic and antitumor activities of cyclooxygenase-2 inhibitors. Cancer Res., 60(5):1306-11, 2000.

Ninomiya, I.; Nagai, N.; Oyama, K.; Hayashi, H.; Tajima, H.; Kitagawa, H.; Fushida, S.; Fujimura, T. \& Ohta, T. Antitumor and anti-metastatic effects of cyclooxygenase- 2 inhibition by celecoxib on human colorectal carcinoma xenografts in nude mouse rectum. Oncol. Rep., 28(3):777-84, 2012.

Labelle, M. \& Hynes, R. O. The initial hours of metastasis: the importance of cooperative host-tumor cell interactions during hematogenous dissemination. Cancer Discov., 2(12):10919, 2012.

Li, W.; Tang, Y. X.; Wan, L.; Cai, J. H. \& Zhang, J. Effects of combining Taxol and cyclooxygenase inhibitors on the angiogenesis and apoptosis in human ovarian cancer xenografts. Oncol. Lett., 5(3):923-8, 2013.

Peng, L.; Zhou, Y.; Wang, Y.; Mou, H. \& Zhao, Q. Prognostic significance of COX-2 immunohistochemical expression in colorectal cancer: a meta-analysis of the literature. PLoS One, 8(3):e58891, 2013.

Perroud H. A.; Rico, M. J.; Alasino, C. M.; Queralt, F.; Mainetti, L. E.; Pezzotto, S. M.; Rozados, V. R. \& Scharovsky, O. G. Safety and therapeutic effect of metronomic chemotherapy with cyclophosphamide and celecoxib in advanced breast cancer patients. Future Oncol., 9(3):451-62, 2013.

Qi, X.; Song, X.; Liu, P.; Yi, T.; Li, S.; Xie, C.; Zheng, Y.; Bai, Y.; Sun, C.; Wei, Y. \& Zhao, X. Antitumor effects of PLGA nanoparticles encapsulating the human PNAS-4 gene combined with cisplatin in ovarian cancer. Oncol. Rep., 26(3):703-10, 2011.

Ragel, B. T.; Jensen, R. L.; Gillespie, D. L.; Prescott, S. M. \& Couldwell, W. T. Celecoxib inhibits meningioma tumor growth in a mouse xenograft model. Cancer, 109(3):588-97, 2007.

Roa, I. Basic Concepts in tumor angiogenesis. Int. J. Med. Surg. Sci., 1(2):129-38, 2014. 
Rosas, C.; Sinning, M.; Ferreira, A.; Fuenzalida, M. \& Lemus, D. Celecoxib decreases growth and angiogenesis and promotes apoptosis in a tumor cell line resistant to chemotherapy. Biol. Res., 47:27, 2014.

Rosas, C. C.; Roa, I.; Sinning O. M.; Fuenzalida B. M. \& Lemus A. D. Celecoxib effect in a multiresistant variant of the TA3 tumor. A histological description. Int. J. Morphol., 31(2):392$8,2013$.

Silverstein, F. E.; Faich, G.; Goldstein, J. L.; Simon, L. S.; Pincus, T.; Whelton, A.; Makuch, R.; Eisen, G.; Agrawal, N. M.; Stenson, W. F.; Burr, A. M.; Zhao, W. W.; Kent, J. D.; Lefkowith, J. B.; Verburg, K. M. \& Geis, G. S. Gastrointestinal toxicity with celecoxib vs nonsteroidal anti-inflammatory drugs for osteoarthritis and rheumatoid arthritis: the CLASS study: A randomized controlled trial. Celecoxib Long-term Arthritis Safety Study. JAMA, 284(10):1247-55, 2000.

Venkatesan, P.; Puvvada, N.; Dash, R.; Prashanth Kumar, B. N.; Sarkar, D.; Azab, B.; Pathak, A.; Kundu, S. C.; Fisher, P. B. \& Mandal, M. The potential of celecoxib-loaded hydroxyapatitechitosan nanocomposite for the treatment of colon cancer. Biomaterials, 32(15):3794-806, 2011.

Vilos, C.; Constandil, L.; Rodas, P. I.; Cantin, M.; Zepeda, K.; Herrera, N. \& Velasquez, L. A. Evaluation of ceftiofur-PHBV microparticles in rats. Drug Des. Devel. Ther., 8:651-66, 2014.

Vilos, C.; Velasquez, L. A.; Rodas, P. I.; Zepeda, K.; Bong, S. J.; Herrera, N.; Cantín, M.; Simon, F. \& Constandil, L. Preclinical development and in vivo efficacy of Ceftiofur-PLGA microparticles. PLoS ONE, 10(4):e0123335, 2015.

Wang, D. \& Dubois, R. N. Eicosanoids and cancer. Nat. Rev. Cancer, 10(3):181-93, 2010.

Wang, X.; Zhang, L.; O'Neill, A.; Bahamon, B.; Alsop, D. C.; Mier, J. W.; Goldberg, S. N.; Signoretti, S.; Atkins, M. B.; Wood, C. G. \& Bhatt, R. S. Cox-2 inhibition enhances the activity of sunitinib in human renal cell carcinoma xenografts. $B r . J$. Cancer, 108(2):319-26, 2013.

Xu, X. F.; Xie, C. G.; Wang, X. P.; Liu, J.; Yu, Y. C.; Hu, H. L. \& Guo, C. Y. Selective inhibition of cyclooxygenase-2 suppresses the growth of pancreatic cancer cells in vitro and in vivo. Tohoku J. Exp. Med., 215(2):149-57, 2008.

Zhang, S.; Da, L.; Yang, X.; Feng, D.; Yin, R.; Li, M.; Zhang, Z.; Jiang, F. \& Xu, L. Celecoxib potentially inhibits metastasis of lung cancer promoted by surgery in mice, via suppression of the PGE2-modulated b-catenin pathway. Toxicol. Lett., 225(2):201-7, 2014.

Zhou, J.; Suzuki, T.; Kovacic, A.; Saito, R.; Miki, Y.; Ishida, T.; Moriya, T.; Simpson, E. R.; Sasano, H. \& Clyne, C. D. Interactions between prostaglandin $\mathrm{E}(2)$, liver receptor homologue-1, and aromatase in breast cancer. Cancer Res., 65(2):657-63, 2005.

\author{
Dirección para correspondencia: \\ Dr. Ignacio Roa Henríquez \\ Unidad de Morfología \\ Departamento de Ciencias Básicas Biomédicas \\ Facultad de Ciencias de la Salud \\ Universidad de Talca \\ Av. Lircay s/n \\ Talca \\ CHILE
}

Email: iroa@utalca.cl

Recibido : 05-02-2015

Aceptado: 15-04-2015 